\title{
COVID-19 emergency department discharges: an outcome study
}

\author{
Authors: David Lanham, ${ }^{\mathrm{A}}$ Jennifer Roe, ${ }^{\mathrm{B}}$ Alisha Chauhan, ${ }^{\mathrm{C}}$ Rebecca Evans, ${ }^{\mathrm{C}}$ Toby Hillman, ${ }^{\mathrm{D}}$ Sarah Logan ${ }^{\mathrm{E}}$ and \\ Melissa Heightman ${ }^{\mathrm{D}}$
}

\begin{abstract}
Pressure on acute medical services in the pandemic mandated an assertive emergency department (ED) discharge policy. Given the potential for subsequent deterioration and growing appreciation of complications relating to COVID-19 infection, this follow up study was instigated to provide clinical reassurance that discharged patients had followed a safe clinical course. 199 patients discharged from the ED of our central London hospital were identified over a 20-day period at the height of the pandemic in April 2020.44 had already reattended ED and 12 had been admitted. At 2-week telephone follow-up, 14 patients were identified who required urgent recall for assessment. At 4-week telephone follow-up, 87 patients were identified with persistent symptoms requiring face to face review. A COVID-19 follow-up clinic was therefore established to provide multi-professional review and diagnostics. 65 patients attended for this assessment. This is the first report on outcomes in COVID-19 infected patients discharged from an ED. It highlights the importance of safety-netting after discharge, the difficulty in predicting which patients might deteriorate and the need for appropriate follow up services.
\end{abstract}

KEYWORDS: COVID-19, ED discharge policy, safety-net, outcomes

DOI: $10.7861 /$ clinmed.2020-0817

\section{Introduction}

The arrival of the COVID-19 pandemic in the UK generated many challenges for the NHS; the most immediate was to prevent hospitals from being overwhelmed by admissions. Strategies were pursued to balance adequate care with the need to maintain

Authors: ${ }^{\text {A }}$ geriatric/acute medicine registrar, University College London Hospitals NHS Foundation Trust, London, UK; ${ }^{\text {Bmicrobiology }}$ consultant, University College London Hospitals NHS Foundation Trust, London, UK; ' Cfoundation year doctor, University College London Hospitals NHS Foundation Trust, London, UK; Drespiratory medicine consultant, University College London Hospitals NHS Foundation Trust, London, UK; Einfectious diseases consultant, University College London Hospitals NHS Foundation Trust, London, UK
What is known?

The clinical profile of hospitalised patients with COVID-19 is now well established but this is the first report on infected patients discharged from ED.

What is the question?

Was our discharge policy too aggressive and what were the early and late complications of the disease after discharge?

What was found?

One in five patients returned to hospital within a fortnight and one in three required review at 6 weeks.

What is the implication for practice now?

A liberal discharge policy is safe provided early hospital review is easily available and a late review clinic can manage patients with persistent symptoms.

bed capacity.This necessitated an active approach to emergency department (ED) discharges. In the face of further waves of infections it is important to evaluate whether these conflicting pressures were resolved satisfactorily.

There have been many reports on disease outcomes from around the world, but this is the first study that follows up patients discharged directly from an ED. Our cohort can be seen as an intermediate severity group of patients (if the most severely affected are those admitted to hospital and the least severe are those who have no hospital contact). We felt that a study of outcomes in this group would be informative in planning future follow up and support strategies.

The standard advice to patients with ongoing illness after ED discharge is either to telephone NHS111 or to contact their general practitioner. However, the pandemic meant that community services were substantially reduced and few doctors were offering face-to-face consultations. Moreover, the NHS 111 call centres were overburdened. ${ }^{1,2}$ As a result, many patients struggled to access timely healthcare advice.

This study had several objectives. What was the clinical course of the patients discharged with COVID-19? Were the discharge criteria adopted at the start of the pandemic appropriate? Could we identify features at initial presentation which increased the risk of reattendance? What was the incidence of long-lasting symptoms and what are the follow-up requirements of this patient group? 
Box 1. The number of patients at discharge meeting our observation criteria

Oxygen saturation $>94 \%(98.5 \%, 196$ out of 199$)$

Heart rate $<110(94 \%, 187$ out of 199)

Respiratory rate $<23(87 \%, 174$ out of 199)

\section{Methods}

Patients presenting with likely COVID-19 infection were assessed for discharge using observation criteria agreed by the Trust (Box 1). Electronic health records were used to identify the 199 patients discharged directly from the University College London Hospital ED between the 1 and 20 April 2020 with a working diagnosis of COVID-19 infection. This was our peak period of COVID-19 attendances and at that stage we felt that urgent review of our strategy was needed. We charted our patients progress at 2, 4 and 6 weeks after discharge (Fig 1).

Demographic data and the following information were recorded: presenting symptoms, vital signs, inflammatory markers, chest X-ray and COVID-19 swab results. When swab results were not available the diagnosis of 'suspected COVID-19 infection' was based on the following criteria: flu-like illness, hypoxia, fever, lymphopenia or typical CXR changes.

Telephone consultations were conducted under consultant supervision by four trainee doctors. An electronic template was built to ensure a standardised approach. Verbal consent was obtained prior to commencing the telephone consultation and patients were deemed to be well if they reported no ongoing symptoms. Observations at presentation and chest $X$-ray findings were noted. Factors indicating increased vulnerability to the virus were recorded: age $>70$ years, living alone and comorbidities. Patient wellbeing was assessed and information on persistent symptoms and breathlessness was noted. If three attempts to make contact failed the patient was noted as uncontactable and their general practitioners was informed if the patient was deemed vulnerable.
The information derived from the 2-week telephone interview led to these allocations:

$>$ no follow-up needed

$>$ telephone review at 4 weeks after discharge

$>$ clinic review with repeat chest X-ray at 6 weeks (for patients with ongoing symptoms and those with previous abnormal chest X-ray)

$>$ urgent review in ambulatory care.

Patients were given a contact number to ring if they had any interim concerns. Because of pandemic pressures decision-making was determined by patient-reported symptoms by telephone rather than direct observation; this may have underestimated symptoms.

Data were analysed on patients selected for clinic review at 6 weeks after discharge. A new COVID-19 follow-up clinic was established for these patients, which also accepted COVID-19 referrals direct from primary care as well as hospital discharges. It offered a one-stop assessment with a consultant physician supervised doctor review, a chest $\mathrm{X}$-ray, blood tests and physiotherapy review. Same day CT thorax imaging and an ECG were performed if clinically indicated. As appointments were scheduled for 6 weeks after ED discharge, Infection Control advised that patients could be seen in a COVID-19 clean area with use of personal protective equipment (masks, gloves, apron) and social distancing. A structured assessment was completed, which included scoring of subjective symptom severity out of 10 at peak illness and on the day of follow-up. There was particular focus on cough, fatigue, breathlessness and sleep quality, as well as GAD2 and $\mathrm{PH} 2$ screening for depression and anxiety.

The respiratory physiotherapist evaluation included a sit to stand exercise desaturation test, where the number of repetitions of standing from sitting with maximal effort using a chair of defined height was recorded. This exercise test has been widely adopted during the pandemic. Breathlessness was scored using the BORG scale and oxygen saturation and heart rate were recorded before and immediately following the minutes testing. The following

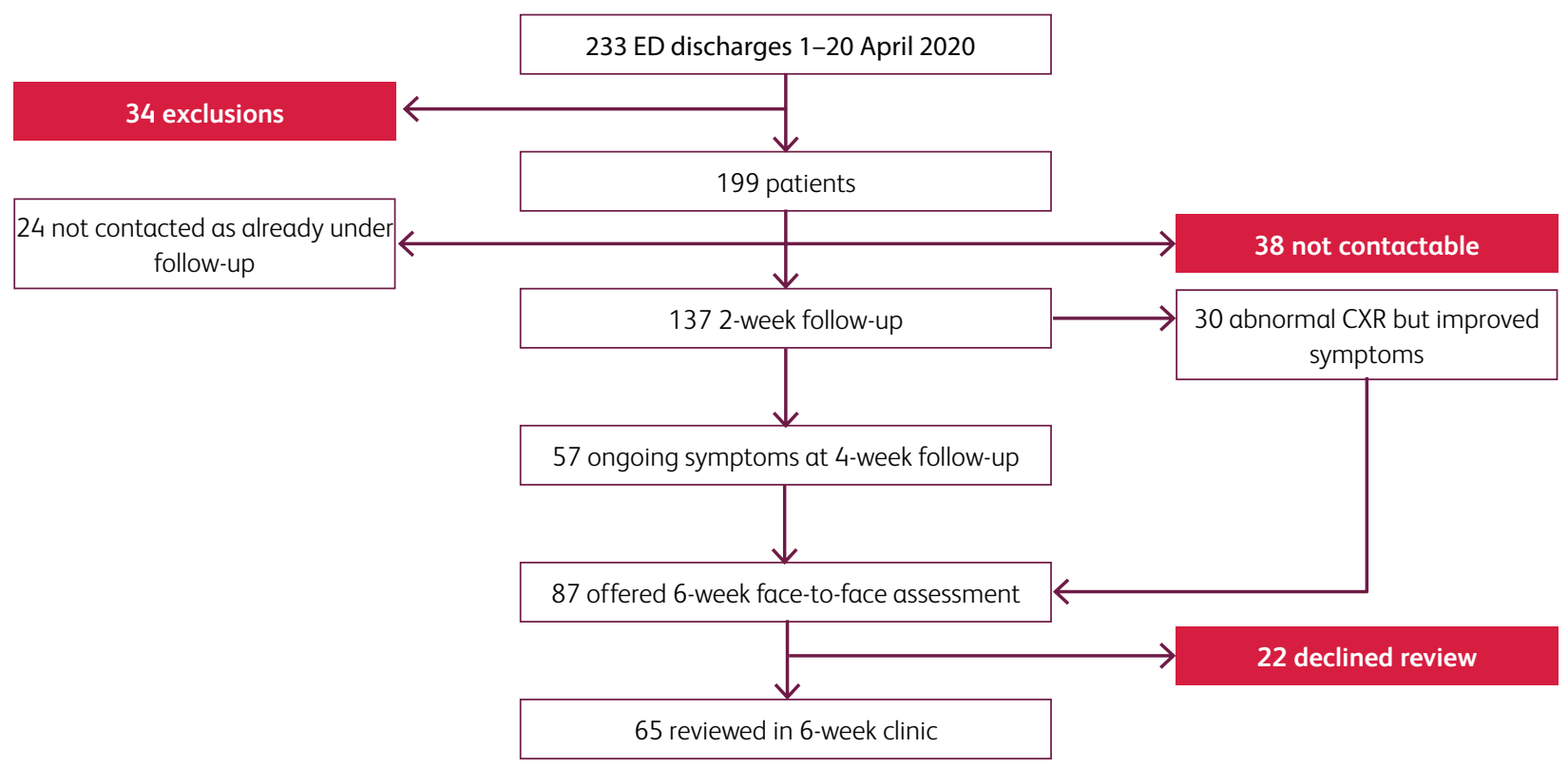

Fig 1. Numbers of patients contacted and reviewed. 
questionnaires were completed where appropriate: the Breathing Pattern Assessment Tool (BPAT), the Fatigue Assessment Scale and the Dyspnoea 12 score.

This service development project was initiated to ensure patient safety and improve quality of care. Therefore ethical approval was not sought, but patients gave verbal consent to telephone consultations. All clinical data were collected as part of standard care and conformed to NHS Health Research Authority guidance.

Microsoft Excel and Graphpad Prism platforms were used for data analysis. Means and standard deviation were reported for parametric data with unpaired t-tests to determine significance and medians/interquartile range and Mann-Whitney tests for significance in non-parametric data. Chi-squared tests were applied to categorical data. COVID-19 PCR testing shortages at this stage of the pandemic resulted in a policy of not testing discharged patients unless there was a doubt about the clinical diagnosis.

\section{Results}

Demographic data

The median age of our cohort of 199 patients was 46, of which $51 \%$ were male. Smoking status was recorded in most patients and 10\% were current smokers. Ethnicity was recorded in $77 \%$ of patients, with $55 \%$ identifying as Black, Asian and minority ethnic (BAME) and $45 \%$ as white. There were 38 patients who could not be contacted by telephone. They had a lower median age at 36 . Two of these patients had been admitted and one had died in another hospital.

\section{Imaging}

The initial chest radiographs were normal in $37 \%$ of patients and 26\% had classic COVID-19 changes (Fig 2). Persistent radiographic changes were seen in $34 \%$ of the 65 patients $X$-rayed at 6 weeks; all but two showed improvement. CT thorax scans were done in seven patients at the 6 -week review due to continuing breathlessness, chest $\mathrm{X}$-ray changes, desaturation on exercise or raised D-dimers. No pulmonary emboli were identified at this stage, but four patients in our cohort had previously been diagnosed with pulmonary emboli. Interstitial lung disease with evolving pulmonary fibrosis was seen in three patients.

\section{Findings in the 6 -week follow-up clinic (65 patients)}

Patients were asked to score a subjective assessment of recovery and $78 \%$ reported that they were more than $70 \%$ recovered (Fig $3 a$ ).

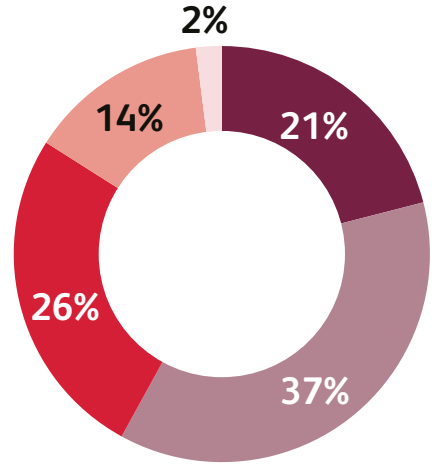

\section{no chest X-ray $\square$ Normal Classic \\ Indeterminate Non-COVID}

Fig 2. Chest X-ray changes at initial presentation to the emergency department.

Many reported severe breathlessness at the peak of their illness (Fig 3b), with subsequent slow improvement. At the 4 -week telephone consultation, $42 \%$ of patients reported persisting breathlessness and this was reported in $43 \%$ in the smaller subgroup seen at 6 weeks. At 6 weeks $78 \%$ reported a more than 2-point improvement in their breathlessness scores but $28 \%$ still had a subjective breathlessness score of $>3$ out of 10 and $22 \%$ had an MRC score of 3 or above (this being a common threshold for referral for pulmonary rehabilitation). There were 10 patients with BPAT scores of 4 or above indicative of a disordered breathing pattern. It is noteworthy that of the seven patients who desaturated on exercise testing at 6 weeks, only one had a subjective breathlessness score above 2 out of 10 . Breathlessness therefore did not correlate directly with recorded hypoxia.

Fatigue was also scored as a severe symptom during peak illness, with two-thirds of patients rating it $\geq 7 / 10$. There was a trend to improvement at 6 weeks but fatigue persisted and was still quite severe in $29 \%$ who had subjective fatigue scores of $>5 / 10$.

Psychological problems troubled $35 \%$ of this cohort, as measured by scoring systems used to assess major depressive disorders; they had PHQ2 or GAD2 scores of 3 or more. These patients were referred to Improving Access to Psychological Therapies (IAPT) services, but two patients required more urgent assessment for suicidal ideation.
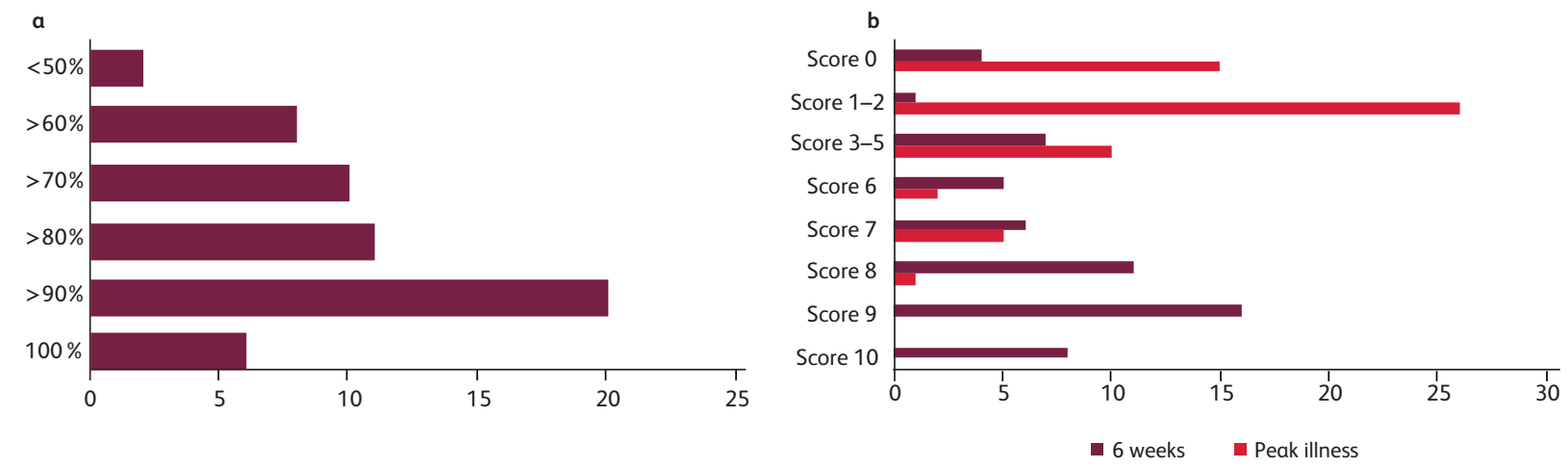

Fig 3. 6-week follow-up graphs. a) Percentage subjective recovery at 6 weeks. b) Breathlessness scores during peak illness and at 6 weeks, 
Assessment of rehabilitation needs revealed that $18 \%$ were still too unwell to work and $32 \%$ could only cope with part-time work. All the patients attending the 6 -week clinic were assessed by a senior respiratory physiotherapist, who felt that $35 \%$ would benefit from a formal rehabilitation programme. The remainder were given written advice on improving their fitness, managing fatigue and returning to work.

Patient feedback was sought in every encounter and all patients found the follow up service useful. Many expressed relief at having a face-to-face review given their difficulty in accessing their general practitioners. Of this group $48 \%$ were of BAME ethnicity compared with $55 \%$ of the whole cohort.

\section{Identifying predictors of reattendance or} adverse outcome

Of our cohort, $22 \%$ reattended and $6 \%$ and needed admission. In addition, $7 \%$ were called back for urgent review in ambulatory care and $44 \%$ were offered in-person review at 6 weeks. Less than a third of all patients sought advice from their GP and only $4 \%$ contacted NHS 111 after their ED attendance.

There were no differences in age or gender between reattenders and non-reattenders. Current or ex-smokers were less likely to reattend. There was a greater proportion of the BAME population in reattending patients ( $61 \%$ vs $39 \%$ ).

Median NEWS score at first presentation was 3 in reattenders and 2 in non-reattenders and median respiratory rate was 24 in reattenders and 22 in non-reattenders (Table 1).

Median CRP at first presentation was significantly higher in patients who reattended ED ( 20.4 versus $10 \mathrm{mg} / \mathrm{L}$ ) and in those admitted compared to those discharged for a second time ( $43.5 \mathrm{vs}$ $4.7 \mathrm{mg} / \mathrm{L}, \mathrm{p}=0.038$ ) (Fig 4); D-dimer was significantly higher in patients who were admitted compared with those did not reattend (2,300 $\mathrm{ng} / \mathrm{mL}$ vs $320 \mathrm{ng} / \mathrm{mL}, \mathrm{p}=0.033)$, but only
Table 1. Median presenting observations in

reattenders and non-reattenders at first emergency

department attendance

$\begin{array}{lll}\text { Median observation } & \begin{array}{l}\text { Reattenders } \\ (\mathbf{1 4 3 )}\end{array} & \begin{array}{l}\text { Non-reattenders } \\ (\mathbf{4 5})\end{array} \\ \text { Pulse } & 93 & 96 \\ \text { Oxygen saturations } & 98 & 99 \\ \text { Respiratory rate } & 22 & 24 \\ \text { NEWS } & 2 & 3 \\ \text { Fever } & 37.2 & 37.4\end{array}$

30 non-reattending patients had a D-dimer checked at first presentation with a median level of $290 \mathrm{ng} / \mathrm{mL}$.

Chest X-ray abnormalities typical of COVID-19 were more common in patients reattending ( $33 \%$ vs $24 \%$ ) and in those subsequently admitted, as well as in those patients with prolonged fever.

Ongoing breathlessness was more prevalent in patients who reattended. Fever for more than seven days at first presentation was more common in reattenders than non-reattenders (56\% versus $34 \%$ ).

\section{Discussion}

Publications from across the world have described the consequences and complications of COVID-19 infection. ${ }^{3}$ Our findings are in keeping with the experience elsewhere in terms of the clinical profile of the disease. But this study is unique in describing the sequelae of patients discharged directly from ED. The symptom burden in this cohort of patients discharged from ED was similar to that observed in hospitalised patients. ${ }^{4}$ Early in the pandemic, few patients with persistent symptoms were

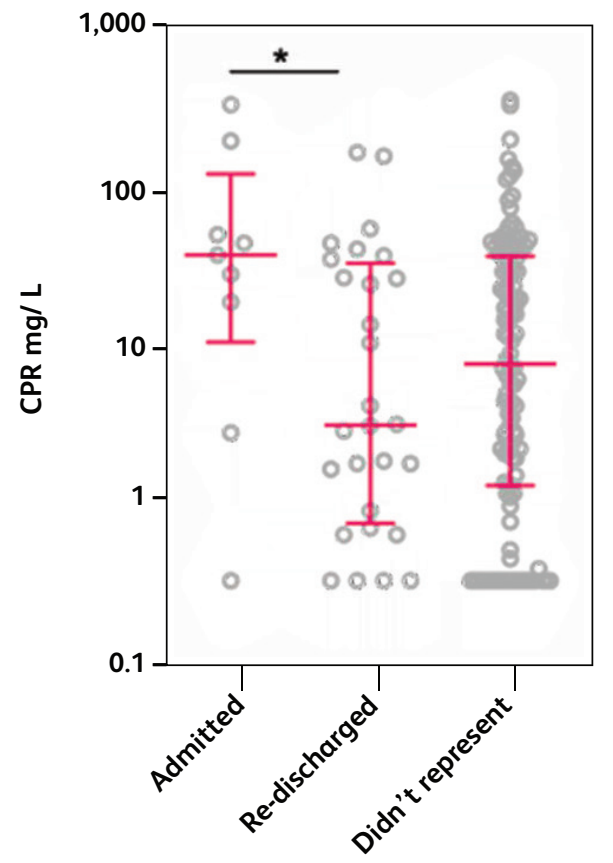

$p=0.0383$

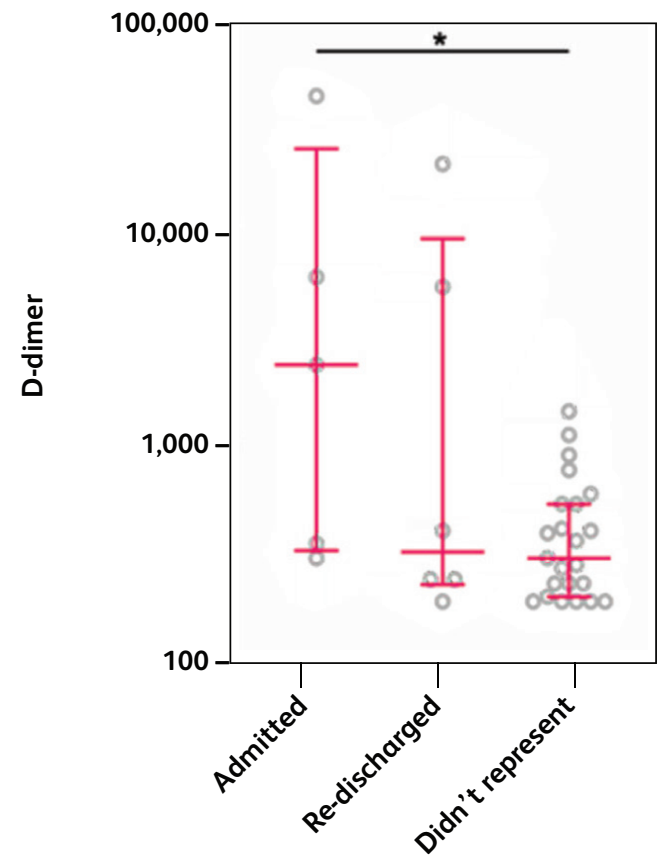

Fig 4. Comparing C-reactive protein and D-dimer in single attendances vs representing to emergency department and subsequently discharged and representing and being admitted. 
offered direct assessment and our service was unusual in providing this. The findings from this study have given us a foundation for the development of formal COVID-19 follow-up services and we have been accepting referrals of community-managed patients since May 2020.

Detailed clinical assessment was only possible in 65 of 87 patients who accepted our invitation for further investigations of persisting symptoms. We have no data on the 22 patients who declined assessment or the 112 patients who had improving or resolved symptoms. In the early stages of the pandemic the importance of ethnicity in determining outcome was not known. But our data do show that the BAME community was overrepresented in patients who reattended or required admission.

Prolonged ill health after the acute infection is now well recognised. ${ }^{5}$ In a cohort of patients with intermediate severity disease, it is worrying that half of the 65 patients seen at the 6 -week review felt too unwell to manage their previous level of employment. In $22 \%$ this was because of breathlessness. In the main, chest radiographic trends were to improvement but there were residual changes in 34\% of those reviewed. Breathlessness and desaturation on testing led to $11 \%$ having a $\mathrm{CT}$ thorax scan. Chronic interstitial lung disease is a concern as a long term consequence of COVID-19 infection but the pandemic is too recent to evaluate this. ${ }^{6}$ Residual interstitial disease was identified in $5 \%$ of patients seen at 6 weeks and the reported 'evolving fibrosis' may have significant implications. A diagnosis of asthma made in $5 \%$, but in many breathlessness was not explained by $\mathrm{CT}$ imaging or echocardiogram and may be attributable to deconditioning or dysfunctional breathing.

The long-term cardiac consequences of the disease are still to be delineated, but a majority of recovering patients have MRI evidence of cardiac involvement.? In this cohort $8 \%$ of patients seen at 6 weeks with continuing breathlessness had raised troponins or abnormal electrocardiograms (ECGs) and are being further investigated. Venous thromboembolism is also widely reported in the early weeks following COVID-19 infection and the incidence was $2 \%$ in our cohort. The D-dimer is often raised in COVID-19 infection and levels can be used to stratify the risk of pulmonary embolism, ${ }^{8}$ but unexpectedly $18 \%$ of patients at 6 weeks had elevated d-dimers at without venous thromboembolism.

Fatigue is a dominant symptom during recovery from COVID-19 and $29 \%$ of patients reviewed at 6 weeks reported this. Fatigue can have multiple causes and there was much overlap between patients suffering with fatigue, breathlessness and psychological distress. The psychological consequences of infection can be considerable and our finding that $35 \%$ suffered from anxiety and depression at 6-week follow-up is consistent with other studies. ${ }^{9}$

The presence of an experienced physiotherapist and an exercise desaturation test during clinic evaluation proved invaluable in assessing breathlessness and providing rehabilitation advice and support. Meeting the significant requirements for rehabilitation will put existing community services under strain as at 6 weeks $35 \%$ of patients were assessed as needing further support.

Good digital record keeping is key to the management of pandemic hospital attendances. Rapid access to all relevant information provides a foundation for all follow-up and safety net planning. Suspected or confirmed COVID-19 cases must be flagged up quickly. Our electronic health record system (EPIC) was an important enabler of this study and delivered the in-house safety net service which was developed for patients discharged from ED following findings reported in this cohort.
Table 2. Prevalence of proposed safety net criteria

in non-reattenders and reattenders

$\begin{array}{lll}\text { Possible risk factor } & \begin{array}{l}\text { No reattendance } \\ (\mathbf{1 5 4 )}\end{array} & \begin{array}{l}\text { Reattenders } \\ (\mathbf{4 5})\end{array} \\ \begin{array}{l}43(30 \%) \\ \text { Lives alone }\end{array} & \begin{array}{l}9(20 \%) \\ \text { Fever }>7 \text { days }\end{array} & 25(56 \%) \\ \mathrm{CRP}>60 & 14(9 \%) & 5(11 \%) \\ \mathrm{O}_{2} \text { saturations } \leq 94 \% & 19(12 \%) & 5(11 \%) \\ <10 \text { days from symptom } & 88(57 \%) & 22(49 \%) \\ \text { onset } & & 17(38 \%) \\ \text { Vulnerable (comorbidities) } & 76(49 \%) & 2(4 \%) \\ \text { Age }>70 & 10(6 \%) & 35(78 \%) \\ \text { Respiratory rate }>22 & 107(70 \%) & \end{array}$

\section{Future safety net and follow-up services}

This study highlights the difficulty in predicting subsequent deterioration and late complications following discharge from ED with suspect COVID-19 infection. The parameters at discharge which suggested a worse outcome were fever for more than 7 days (Table 2) and high CRP and D-dimer levels (Fig 4). These findings are similar to a UK study of readmission of hospitalised patients ${ }^{10}$ but patient numbers are too low to identify thresholds for these parameters. This is being elucidated by the ISARIC study. ${ }^{11}$ Provisional results suggests the $4 \mathrm{C}$ Mortality Score may prove a useful predictor of mortality at ED presentation..$^{12}$ Our study showed a median NEWS2 score of 3 in reattenders compared to A median NEWS2 score 2 in patients who did reattend. However, a larger study revealed NEWS2 to have poor-to-moderate discrimination for medium term COVID-19 outcome. ${ }^{13}$

While we have demonstrated the success of a 2 -week telephone review of all ED-discharged patients, many organisations lack the resources to deliver this, particularly when acute services are under pressure. An easily accessible hospital telephone hotline could be an alternative, but there is growing support for community remote monitoring programs under primary care. There is a need to ensure follow-up services adequately meet the needs of BAME patients and our 6 -week review figures only indicate a modest underrepresentation of this group.

Unsurprisingly, chest X-ray abnormalities at presentation were more common in patients who reattended, but it is noteworthy that a third of patients who subsequently required admission had a normal initial chest X-ray. The first X-ray may not have demonstrated changes at an early stage of their illness. Telephone consultations have been widely employed during the pandemic, but our experience indicates that assessing breathlessness by telephone consultation is challenging and requires careful question design. Concurrent home oximetry would significantly improve the quality of the assessment, particularly given the poor association between desaturation and subjective experience of breathlessness in these patients. This could be augmented by video consultation which might include home assessment of desaturation on exercise by sit to stand testing.

Our 6-week clinic proved invaluable in the management of patients with complications and provided reassurance and guidance for individuals with a range of chronic post-COVID-19 symptoms. 
The British Thoracic Society advises that chest radiography be done 3 months after discharge in all patients admitted with COVID-19 infection. ${ }^{14}$ Our finding of residual radiographic abnormalities in so many patients at 6 weeks lends support for this proposal, but our experience with other symptoms suggests that earlier follow-up is preferable. Our multispeciality, multiprofessional COVID-19 followup service has booked over a thousand appointments with referrals coming from primary care, ED discharges and admission discharges. In-person assessment with access to hospital diagnostics is essential for adequate evaluation. The rehabilitation needs and need for psychological support in this group is considerable; to deliver this in a timely and equitable fashion will require a significant reconfiguration of services.

\section{Conclusion}

This study shows how the need to keep hospital beds available during the pandemic resulted in the discharge of many patients from ED who subsequently deteriorated or failed to recover. However, it suggests that a more liberal admission policy is unnecessary if there is adequate follow-up. It shows how difficult it is to identify those patients most likely to deteriorate and argues for better criteria to identify at risk individuals. We demonstrate the importance of an augmented safety net service to pick up early deterioration, especially respiratory failure and venous thromboembolism. There is also a need for review clinics for patients with ongoing symptoms. We discuss these safety net and follow-up services.

\section{References}

1 Leading Healthcare. $77.8 \%$ of NHS 111 calls NOT advised to go to Emergency Department. www.leadinghealthcare. co.uk/2020/03/11/77-8-of-nhs-111-calls-not-advised-to-go-toemergency-department/.

2 Dalton J. Callers to NHS 111 phone line wait hours and get cut off without being able to speak to nurse. Independent, 13 March 2020. www.independent.co.uk/news/uk/home-news/coronavirusuk-symptoms-nhs-111-phone-line-nurse-a9400351.html.
3 Esakandari H, Nabi-Afjadi M, Fakkari-Afjadi ] et al. A comprehensive review of COVID-19 characteristics. BiolProced Online 2020;22:19.

4 Halpin SJ, McIvor C, Whyatt $\mathrm{G}$ et al. Post discharge symptoms and rehabilitation needs in survivors of COVID-19 infection: A crosssectional evaluation. J Med Virol 2020, in press (doi: 10.1002/ jmv.26368).

5 National Institute for Health and Care Excellence. Management of the long-term effects of COVID-19. NICE guideline [NG188]. NICE, 2020. www.nice.org.uk/guidance/gid-ng10179.

6 Fraser E. Long term respiratory complications of COVID-19. BM] 2020;370:m3001.

7 Puntmann VO, Carerj ML, Wieters I et al. Outcomes of cardiovascular magnetic resonance imaging in patients recently recovered from coronavirus disease 2019 (COVID-19). JAMA Cardiol 2020;5:1265-73.

8 Kaminetzky M, Moore W, Fansiwala K et al. Pulmonary embolism on CTPA in COVID-19 Patients. Radiol Cardiothorac Imaging 2020;2:e200308.

9 Mazza MG, Lorenzo RD, Conte $\mathrm{C}$ et al. Anxiety and depression in COVID-19 survivors: role of inflammatory and clinical predictors. Brain Behav Immun 2020;89:594-600.

10 Rokadiya S, Gil E, Herbert R et al. Outcomes of confirmed COVID-19 re-admitted to hospital. J Infect 2020;81:e18-e19.

11 Docherty A, Marrison EM, Green CA et al. Features of 20133 UK patients in hospital with covid-19 using the ISARIC WHO Clinical Characterisation Protocol: prospective observational cohort study. BMJ 2020;369:m1985.

12 Knight SR, Ho A, Pius R et al. Risk stratification of patients admitted to hospital with covid-19 using the ISARIC WHO Clinical Characterisation Protocol: development and validation of the $4 \mathrm{C}$ Mortality Score. BMJ 2020;370:m3339.

13 Carr E, Bendayan R, Bean D et al. Evaluation and Improvement of the National Early Warning Score (NEWS2) for COVID-19: a multihospital study. medrxiv 2020 (doi: 10.1101/2020.07.30.20165464v1).

14 British Thoracic Society. Guidance on respiratory follow-up of patients with a clinic-pathological diagnosis of covid-19 pneumonia. BTS, 2020.

Address for correspondence: Dr David Lanham, University College Hospital, 235 Euston Road, London NW1 2BU, UK. Email: david.Ianham@nhs.net 